Populations vulnérables

\title{
L'expérience plurielle de la vulnérabilité au sein des bidonvilles
}

The plural experience of vulnerability in slums

Jean-Baptiste Daubeuf

\section{OpenEdition}

1 Journals

Édition électronique

URL : https://journals.openedition.org/popvuln/1032

DOI : 10.4000/popvuln.1032

ISSN : 2650-7684

Éditeur

LIR3S - Laboratoire Interdisciplinaire de Recherche "Sociétés Sensibilités Soin" (UMR 7366 CNRS-uB)

\section{Édition imprimée}

Date de publication : 1 mars 2019

Pagination : 79-96

ISBN : 978-2-918173-26-7

ISSN : 2269-0182

Référence électronique

Jean-Baptiste Daubeuf, « L'expérience plurielle de la vulnérabilité au sein des bidonvilles », Populations vulnérables [En ligne], 5 | 2019, mis en ligne le 26 mai 2020, consulté le 26 février 2022. URL : http:// journals.openedition.org/popvuln/1032 ; DOI : https://doi.org/10.4000/popvuln.1032

Ce document a été généré automatiquement le 26 février 2022.

Les contenus de la revue Populations vulnérables sont mis à disposition selon les termes de la Licence Creative Commons Attribution 4.0 International 


\section{L'expérience plurielle de la vulnérabilité au sein des bidonvilles}

The plural experience of vulnerability in slums

Jean-Baptiste Daubeuf

\section{Introduction}

1 La Fondation Abbé Pierre considère qu'il y aurait aujourd'hui près de 15 millions de personnes en France dans des situations de vulnérabilité face au logement. Parmi eux, on compterait 4 millions de mal logées, autrement dit des personnes "privées de logement ou vivant dans des conditions très difficiles " (Fondation Abbé Pierre, 2018). À ce chiffre, il faudrait également ajouter les 12 millions de locataires fragilisés dans leur rapport au logement (copropriété difficile, loyers ou charges impayées, surpeuplement modéré, précarité énergétique ou effort financier excessif). Le phénomène du mallogement est donc massif, mais il est également très hétérogène, aussi bien en termes de conditions de précarité que de populations concernées.

Parmi l'ensemble des situations de mal-logement en France, les bidonvilles en représentent probablement l'une des expressions les plus visibles et médiatiques. Selon les chiffres produits par la Délégation interministérielle à l'hébergement et à l'accès au logement (DIHAL), environ 15000 personnes pour la majorité originaires d'Europe de l'Est et identifiées comme Roms, réparties dans un peu plus de 500 installations, vivraient aujourd'hui dans ce type d'habitat en France métropolitaine (DIHAL, 2017). À ces installations, il faut ajouter les campements de migrants de la région parisienne ou à la frontière entre la France et le Royaume-Uni ainsi que les bidonvilles d'outremer, probablement au moins deux à trois fois plus nombreux qu'en métropole (Damon, 2017). Même si les phénomènes se sont transformés et connaissent des dynamiques différentes, les chiffres ont très peu évolué depuis une dizaine d'années. Tandis que les bidonvilles de métropole liés aux migrations d'Europe de l'Est se stabilisent et parfois décroissent, au contraire les campements de migrants, dont les regroupements peuvent mener à des installations collectives de plusieurs milliers d'occupants, se multiplient et 
apparaissent ponctuellement en fonction de l'intensité et de la régularité des expulsions. À mesure qu'ils se stabilisent, ces campements se rapprochent alors des conditions de vie que l'on rencontre dans les bidonvilles.

3 Ainsi, à l'image de la précarité résidentielle identifiée par la Fondation Abbé Pierre, le phénomène des bidonvilles découle de multiples facteurs. Il est tout à la fois le résultat d'un manque structurel de logements, d'une absence de prise en charge sociale et institutionnelle, d'une stigmatisation des habitants ainsi que de politiques de l'État qui participent à l'accroissement de leur fragilité, forçant et contraignant certaines populations à se replier sur ces formes précaires d'habitat. Il s'agit donc en partie d'un habitat subi, conduisant leurs occupants à endurer des conditions matérielles et sociales éprouvantes, les enfermant parfois au fil du temps dans une situation de grande pauvreté.

4 Toutefois, face à l'ordre urbain qui s'impose à eux, les habitants des bidonvilles ne sont jamais complètement soumis et passifs (Certeau, 1990). Loin d'être démunis face à la pauvreté, ils déploient de multiples tactiques et stratégies dans l'optique d'accéder à des prises spatiales et sociales, aussi fragiles soient-elles, afin de minimiser l'impact des contraintes extérieures sur leur existence. À travers des interstices dans les marges urbaines (Gaulejac, Blondel et Taboada-Leonetti, 2014 ; Lussault, 2009), ils aménagent des ancrages précaires pour accéder à la ville, fixer leur foyer à un lieu et faire ainsi perdurer leur vie au sein d'un même espace. C'est pourquoi les bidonvilles sont en partie le résultat de micro-résistances face à l'ordre urbain (Scott, 2009) de la part de populations qui disposent, malgré tout, de ressources pour négocier leur présence en ville (Lion, 2015). Ainsi, les habitants des bidonvilles personnalisent la forme que prennent leurs ancrages précaires, différemment, par exemple, des micro-espaces occupés dans les interstices urbains ou des logements squattés par certaines des autres populations confrontées à la vulnérabilité résidentielle (Girola, 2011 ; Pichon, 2010).

5 Les bidonvilles ne sont donc pas un habitat précaire comme les autres. Tout du moins, ils possèdent des particularités qui leur sont propres. Ils sont le résultat d'agencements à l'interface de contraintes structurelles et de résistances locales. Indirectement, donc, la fragilité que connaissent leurs habitants ne s'interroge pas seulement par le haut, mais également par le bas, autrement dit par la manière dont ils «amortissent » et donnent sens à la vulnérabilité (Martuccelli, 2006).

6 Cet article propose de comprendre ce qui caractérise l'expérience de la vulnérabilité résidentielle en interrogeant la multiplicité des formes qu'elles peuvent prendre dans les bidonvilles. Il s'agit ainsi de distinguer les expériences vécues par les habitants en allant voir au plus près de la vie sur place, les différences en termes de parcours migratoires, de ressources économiques, d'habitat et de relation à la société française.

7 Nous nous appuierons sur un travail ethnographique mené au sein du bidonville de « La place ", dans le quartier du Haut-du-Lièvre, à Maxéville, en banlieue de l'agglomération nancéienne, dans l'Est de la France, entre 2014 et 2016. C'est dans ce bidonville que séjournait alors une cinquante de familles originaires de Bărbulești, village du Sud de la Roumanie. Nous avons suivi leur quotidien pour recueillir aussi bien le «dire " que le "faire» et ainsi comprendre les différentes "épreuves" (Martuccelli, 2010) qui y structurent le quotidien. Une ethnographie au long cours a été menée dans le but de saisir les principales épreuves (la migration, l'économie urbaine, l'habitat, la race ou le genre) ainsi que les formes de vie qu'induit le bidonville. Pour autant, si ce travail de 
terrain a permis d'appréhender les modalités sociales spécifiques à ce type d'habitat, il a aussi montré l'hétérogénéité des parcours et des formes d'appropriation de l'espace.

\section{Des parcours menant à des expériences plus ou moins marquées de la vulnérabilité}

Pour les migrants roumains les moins dotés en ressources, leur installation en ville s'inscrit dans des parcours sur le temps long (Potot, 2007). Le bidonville constitue un lieu temporaire d'implantation, représentant parfois une amélioration des conditions d'habitat, à d'autres moments une régression. Dès lors, il serait faux de croire que les parcours qui y mènent se ressemblent tous. Bien au contraire, les périodes ayant généralement suivi l'arrivée des migrants en France, avant leur installation dans le bidonville, les ont amenés à connaître des conditions de vie très variables. Surtout, ces parcours migratoires ne sont pas unidirectionnels. Au contraire, ils dépendent d'allersretours entre le pays d'origine et le lieu d'arrivée (Sayad, 2014), de périodes de grandes mobilités ou de confrontations avec les pouvoirs publics (Agier, 2014). Ainsi, tandis que pour certains le bidonville favorise une autonomisation vis-à-vis de leur pays de départ, au contraire, pour d'autres, il maintient, parfois accentue, la dépendance des habitants à l'ordre social traditionnel.

9 La situation de fragilité rencontrée lors des parcours migratoires fait alors souvent écho aux difficultés rencontrées par certaines familles au pays. Dans le cas des habitants de "La place », revenir vivre dans le village représente généralement un objectif plus ou moins primordial et atteignable. Pour les plus pauvres, les ressources peu nombreuses dont ils disposent leur laissent difficilement la possibilité de se projeter dans un avenir clair à Bărbulești alors que l'emploi est absent ou faiblement rémunérateur. Surtout, ils continuent d'être confrontés au village à des situations de dépendance et d'obligations contraintes vis-à-vis de certains représentants locaux de la mafia, ce qui peut les maintenir dans des formes aiguës de captivité, voire d'asservissement (Bourgois et al., 2015). En outre, les migrants bénéficient en Roumanie de logements allant d'édifices en quasi-ruines jusqu'à des constructions disposant de tout le confort moderne. Dès lors, la fragilité des liens qui rattache les habitants à la Roumanie et leur parcours en ville sont marqués de façons différenciées par l'incertitude de savoir où se réfugier en cas de problème.

À «La place », on observe des trajectoires migratoires différentes sous de nombreux aspects. Tandis qu'une partie des migrants ont débuté leur séjour en Europe de l'Ouest depuis plus de 25 ans, d'autres au contraire ne sont arrivés que depuis quelques années seulement. Ainsi, parmi les hommes du bidonville, plusieurs ont fait leur premier séjour en Allemagne ou en France dès 1990. C'est par exemple le cas d'Ion qui a vécu quelque temps dans un bidonville à Bobigny juste après la chute du mur de Berlin :

Avec quelques copains, on avait ouvert un platz là-bas. On ne trouvait nulle part où aller. Paris est une ville qui fait peur parce que c'est grand et dangereux. Certains gars de Bărbulești avaient déjà fait ça et on les avait laissés tranquilles pendant plus d'un an. Alors on s'est réuni à 15-20 et on a commencé à construire des cabanes sous un pont. Au début, la police nous a rien dit. Mais après ils ont commencé à être là souvent et à nous demander nos papiers. Moi je suis rentré en Roumanie pour m'occuper de ma femme et de mes enfants. Pas très longtemps après le platz a été démoli par la police. 
11 Durant cette période, la mobilité des habitants est liée en partie à la fragilité de leur inscription spatiale en ville. Leur quotidien était alors essentiellement rythmé par une alternance d'emménagements temporaires et de départs rapides, qu'ils soient contraints par des expulsions ou animés par l'envie d'aller vers des lieux économiquement plus prospères. C'est ce qu'a connu ainsi Adi, 28 ans au moment de notre enquête et père de deux filles. Il a quitté la Roumanie à 14 ans pour suivre des cousins en France. Son installation dans des lieux en "durs » n'est pour lui qu'une donnée récente puisque pendant de longues années, il a principalement circulé de ville en ville à la recherche d'activités lucratives. Ainsi, les différentes expériences qu'il a vécues font que le bidonville représente dans son cas une amélioration claire de ses conditions de vie:

J'ai tout connu, la rue, la voiture, les ponts, les caravanes même les hôtels, depuis que je fais ça. Pendant deux ans on a fait le "tour de France ", j'ai fait Blois, Dunkerque, Montpellier... On passait dans les platz des gens de Bărbuleș̦i et le plus souvent on dormait dans la rue, sur des cartons, serrés tous ensemble. Si on arrivait à faire suffisamment d'argent, on se payait une chambre d'hôtel. Parfois, c'était des gens gentils qui me voyaient et qui me disaient «tiens je te paye l'hôtel». Quand il faisait trop froid, j'allais dans les commissariats, je mettais ma couverture sur le banc et je dormais là sans rien dire à personne. J'étais mineur. Ils étaient obligés de me laisser au chaud. Je leur disais «monsieur, vous ne pouvez pas me mettre dehors, je suis mineur». Des fois, les policiers appelaient les foyers et ils me trouvaient une chambre. Le plus souvent, ils ne voulaient pas s'emmerder alors ils me laissaient dormir dans le hall du commissariat.

Néanmoins, pour la majorité des familles du bidonville de « La place », leur parcours en ville s'accompagne d'une crainte de se retrouver encore une fois à la rue. Les années dans le froid constituent un souvenir marqué dans les corps et certains habitants en portent encore les marques (apparence prématurée de vieillesse, problèmes respiratoires, dentaires ou dermatologiques).

Les femmes et les enfants ne sont pas restés à l'écart de ces mouvements migratoires et parmi eux plusieurs ont suivi leur conjoint ou leur père rapidement après les premiers départs vers l'Europe de l'Ouest. Malgré tout, jusqu'à la fin des années 2000 et la stabilisation des familles dans l'agglomération de Nancy, la forte mobilité a limité la présence des plus fragiles. C'est pourquoi la majorité d'entre eux sont arrivés depuis moins de dix ans. Cette hétérogénéité des parcours mène les habitants du bidonville à porter des regards différents sur leurs conditions de vie. C'est ce qu'explique Viorel, 26 ans, père de famille d'un garçon de 2 ans. Lorsqu'il était plus jeune, il a connu les mêmes conditions qu'a décrites Adi. Il voit dans les plaintes de certains de ses voisins un manque d'endurance et d'expériences :

Les familles ici, elles ont peur pour n'importe quoi. Elles ont pas connu la rue alors qu'avant c'était carrément pire, même dans le quartier du Haut-du-Lièvre, il y avait de la drogue, des armes, des bagarres. Quand t'as vécu comme moi la misère, tu sais qu'ici c'est rien. Tu sais qu'on va pas te dégager sans te prévenir, que demain tu vas manger. Je préférerais un appartement pour mon fils, mais en attendant ça reste tranquille. Il faut arrêter de pleurer.

14 Ici, donc, la vulnérabilité ne semble pas s'inscrire dans le cadre d'une condition commune, mais bien plutôt dans une multiplicité de parcours donnant une signification à chaque fois différente à l'expérience du bidonville. 


\section{Des fragilités économiques hétérogènes}

La vulnérabilité liée aux revenus pèse très lourdement sur le quotidien des familles du bidonville. C'est notamment pour cette raison que les habitants multiplient les activités économiques. Ces dernières sont de différentes natures : la récupération de métaux ou d'objets pouvant être revendus, le petit commerce de fleurs durant le printemps, la mendicité, le «travail au noir ». Dès lors, la fragilité monétaire des habitants de «La place » dépend principalement du domaine économique au sein duquel ils s'inscrivent, de leur flexibilité pour passer de l'une ou l'autre de ces activités et de la solidité des compétences qu'ils ont développées au cours du temps. Cet ensemble d'éléments montre finalement l'existence d'une hétérogénéité d'expériences de la vulnérabilité économique au sein du bidonville, qui peut être caractérisée en fonction de l'incertitude des revenus générés et des risques judiciaires encourus.

Parallèlement à cela, il apparaît nécessaire de voir qu'un ensemble de phénomènes s'exerçant à des échelons bien supérieurs à ceux du quartier transforment quotidiennement l'environnement économique de «La place» : évolutions des cours mondiaux des métaux, changements législatifs relatifs à l'encadrement du travail, arrêtés municipaux portant sur la pratique de la mendicité, mouvements migratoires accentuant la concurrence au sein de certains domaines d'activités, etc. La stabilisation des revenus représente alors un enjeu primordial pour les habitants, ce qui leur demande parfois des investissements lourds, comme l'achat de matériel roulant pour le glanage de métaux ou la récolte de fleurs. À cela s'ajoute aussi très souvent la nécessité de rogner sur les revenus pour faire face à la concurrence, notamment dans le cas du travail au noir ou de la vente d'objets de récupération.

Une variation des cours mondiaux du cuivre ou de l'aluminium peut avoir plusieurs types d'incidences et provoquer des pertes de revenus de différentes manières. En effet, d'un côté, un cours trop bas remet en question les investissements effectués et laisse en difficulté le ferrailleur pour rembourser son matériel ou ses outils. De l'autre, un cours trop haut peut multiplier la concurrence, générer une recrudescence des vols sur les chantiers ou les bâtiments abandonnés et par répercussion augmenter les contrôles policiers ou les opérations de répression.

On trouve le même type d'exemple dans le cas de la mendicité dont les revenus générés par les habitants qui se prêtent à cette activité semblent avoir été largement influencés par les controverses portant sur la question rom. Alors qu'avant cette période, pour certains d'entre eux les revenus pouvaient parfois dépasser $300 €$ pour une semaine de mendicité, aujourd'hui ils arrivent, selon eux, difficilement à atteindre les $150 €$. Même pour ce type d'activité donc, des facteurs extérieurs peuvent peser de façon très contraignante sur les habitants du bidonville. Dès lors, pour les habitants de «La place ", les risques sont grands de voir leurs revenus fortement décroître à certaines périodes sans pouvoir anticiper celles-ci.

19 Un deuxième facteur de vulnérabilité économique découle de la proximité de ces activités avec la limite, parfois floue, entre le légal et l'illégal. La forte concurrence pesant au sein des domaines les plus rémunérateurs pousse ou a poussé un certain nombre d'habitants à transgresser parfois la frontière du droit, en particulier dans le cas de la récupération de ferraille. En effet, la raréfaction des métaux et l'attrait monétaire généré par l'augmentation de leur cours ont été des éléments déclencheurs pour certains jeunes hommes du bidonville, les amenant à multiplier les actions de vol 
de cuivre sur des chantiers ou dans des propriétés privées. De la même manière, pour augmenter leurs revenus, certains mendiants pratiquent ce qui peut être considéré comme des formes de mendicité agressive. Ils insistent alors auprès des passants pour obtenir de l'argent, en passant parfois par des moyens détournés comme dans le cas des formulaires de sourds et muets distribués à des piétons qui sont ensuite suivis et harcelés. Ce dernier type de mendicité a amené l'agglomération à réprimer fortement ceux qui les pratiquent et plusieurs habitants de «La place» ont écopé de peines pouvant aller jusqu'à plusieurs mois de prison avec sursis.

Encore une fois, la vulnérabilité des familles dépend en grande partie de leur situation.

Ainsi, certaines d'entre elles bénéficient de situations plus favorables que les autres. Parmi les ferrailleurs, le partage du travail au sein des groupes d'hommes d'une même famille facilite le glanage et étend la zone d'action. Les lieux de collectes ont également été en partie répartis et chacun dispose par exemple d'une déchetterie qui lui est attitrée.

21 Ces derniers éléments montrent que les parcours et les activités des habitants dépendent largement de que Nicolas Renahy et Jean-Noël Retière nomme le «capital d'autochtonie » (Renahy, 2010 ; Retière, 2003). Ce dernier facilite l'accès des familles à des revenus stabilisés, voire permanents, et il joue un rôle prépondérant dans la mise en forme de l'expérience de la vulnérabilité économique. Ce capital leur permet notamment de se faire en partie respecter dans le monde des mendiants au sein duquel la concurrence s'exerce en permanence et les places les plus rémunératrices font l'objet de prédation violente. Dès lors, en fonction des événements pouvant remettre en question certaines sources de revenus, les habitants connaissent des périodes d'incertitude plus ou moins régulières ou fortes.

\section{Une prégnance variable de la promiscuité}

22 À cela s'ajoute une autre variable, relative au confort, à l'espace ou à la qualité des matériaux. Ces différences s'expliquent de plusieurs manières. Ainsi, lors de leur installation, certaines familles ont pu obtenir de l'aide d'habitants du quartier, de travailleurs sociaux et de bénévoles ou bénéficier de dons de matériaux leur permettant d'élaborer des constructions de meilleure qualité, mieux chauffées, isolées, aérées et lumineuses. En outre, les conditions économiques des habitants au moment de leur installation étaient hétérogènes et certains ont bénéficié de ressources leur permettant d'investir dans des caravanes en meilleur état. Parce que la majorité des habitations mobiles présentes sur le bidonville sont âgées, elles connaissent des défaillances plus ou moins importantes et la construction de cabanes résulte en partie des difficultés auxquelles ont été confrontées certaines familles pour acquérir une caravane habitable. Pour preuve, Adi, jeune homme auquel nous avons déjà fait référence un peu plus haut, nous explique ainsi que :

c'est seulement quand le maire nous a dit qu'on pouvait s'installer qu'on a construit des cabanes. Avant il n'y avait pas de cabane. C'est mon père qui a commencé. On avait plus d'argent, on était très pauvres à ce moment-là, alors il nous a laissé Remus et moi dans les caravanes et il a construit une cabane pour ma mère et mes frères. Après on a construit la cuisine et des cabanes pour mettre des trucs. Ensuite, toute «La place» a fait pareil. C'est pour ça que tout le monde a des cabanes maintenant. Après ça, on a construit les deux cabanes derrière pour mettre les vêtements et des outils. Celles-là, c'est pas des vraies cabanes parce qu'on a pas fait 
les murs biens. Nous, on est ceux qui savent le mieux construire des cabanes. Celle de mon père elle est bien, elle est grande et la chaleur elle part pas trop vite. Maintenant, il n'y a que Florin et ştefania qui ont aussi une cabane, mais elle n'est pas aussi bien même si ça va. Avant tu aurais vu les autres, ils avaient des cabanes, c'était vraiment la misère...

23

La qualité des constructions pèse donc sur le quotidien. Dans certaines baraques, les familles ne disposent que de poêles à bois qui nécessitent de collecter du bois en ville et le découper chaque soir à la hache. D'autres habitations au contraire bénéficient de poêles à pétrole dont le combustible est fourni par une association, mais dont le principal désavantage est de produire une chaleur asséchant les muqueuses et piquant les yeux. En outre, la taille des groupes familiaux joue sur l'importance des tâches quotidiennes au sein des foyers. Plus les familles sont nombreuses, plus le fonctionnement interne des maisonnées se complique. C'est en particulier le cas de l'approvisionnement en eau dont le point d'accès le plus proche se trouve à plusieurs centaines de mètres et nécessite ainsi d'effectuer des allers-retours munis de gros bidons tractés sur des caddys. Cette tâche, en plus de geler les mains en hiver, demande de porter à bout de bras des charges lourdes alors que ce sont principalement les enfants ou les femmes qui doivent s'en charger. Pour les familles les plus nombreuses, il est alors nécessaire de faire plusieurs trajets par jour. Ceci est d'autant plus indispensable que les lessives s'effectuent à la main et que la multiplication du nombre de membres au sein d'un foyer a une répercussion directe sur la masse de linge sale.

À ces problèmes s'ajoute également la nécessité de nourrir les membres du foyer. Là encore, les tâches sont d'importance variable selon les groupes familiaux et demandent à certaines femmes d'y consacrer l'ensemble de leur temps en journée, là où d'autres au contraire y passent seulement quelques heures. On retrouve la même situation concernant l'entretien des habitations. Ainsi, d'un foyer à l'autre, le temps nécessaire pour le ménage varie fortement. Chez les plus anciens, comme le couple Grigore et Camelia Gîngioveanu, 61 et 60 ans ou chez Nicolae et Ilena Cîrciu, 56 et 55 ans, le temps à s'occuper de leur lieu de vie se limite à moins d'une heure ou deux le soir après avoir passé la journée en centre-ville pour mendier. Au contraire, chez Ion et Vicotria Cîrciu ou chez Paul et Christina Băsescu, les deux plus grands groupes familiaux, le ménage, les lessives, la préparation des repas mobilisent les femmes une grande partie de la journée. Le nombre d'enfants, le caractère salissant des activités économiques ou encore des habitations généralement en moins bon état, obligent les mères de famille à passer de longs moments à nettoyer les sols, récupérer les ustensiles de cuisine ou lessiver les vêtements.

25 La taille des groupes familiaux a d'autres répercussions. Alors que plusieurs couples sont isolés et mène une vie relativement indépendante, d'autres au contraire sont confrontés à la promiscuité et la proximité des corps. Certaines familles disposent d'un espace domestique relativement important, ce qui leur offre une autonomie forte, tandis que d'autres bénéficient seulement de très petits espaces et sont contraints de vivre en contact constant les uns avec les autres. Cette promiscuité pèse sur la possibilité de chacun des membres de la famille à s'approprier un espace personnel et à maintenir un lieu d'intimité qui ne soit pas envahi par la collectivité.

Ainsi, en fonction des familles, l'espace par habitant peut aller du simple au quadruple. Mais surtout, il peut être plus ou moins partagé. Par exemple, Christi, 18 ans et vivant avec ses parents, dispose d'une petite cabane pour lui qu'il a pu investir en y ajoutant au mur des éléments de décoration ou simplement en se retrouvant seul. Plusieurs

Populations vulnérables, 5 | 2019 
adolescents au contraire, comme Mihai et Constantin, respectivement 15 et 17 ans, se retrouvent dans l'obligation de partager leur lit dans la même cabane que celle de leurs parents et de leur petit frère Virgil, 8 ans. La vulnérabilité relative de l'espace du " chez-soi » montre comment, selon les situations de chacun, la signification de la notion de l'habiter prend des formes profondément différentes au sein du bidonville.

\section{Des rapports aux institutions et à la société française multiples}

Comme nous l'avons vu, la présence en France des habitants de «La place » est plus ou moins ancienne. Ils ont ainsi eu l'occasion d'appréhender de façon relativement différente les codes et les normes de la société française. Surtout, ils ont connu les évolutions du stigmate associé à l'identité rom. En effet, dès 2010, pendant le mandat de Nicolas Sarkozy comme président de la République, les occupants de ce qui est alors désigné par le terme de «campement» ont fait l'objet de politiques massives d'expulsions visant à réprimer les installations illégales. Si cette campagne politicomédiatique ne visait alors pas encore directement les migrants d'Europe de l'Est, elle a été un moment déclencheur de la dégradation de l'image des Roms et de leur association avec le problème de l'augmentation de l'insécurité au sein de l'Hexagone. À partir de 2012, Manuel Valls, alors premier ministre, a réinvesti cette thématique en s'engageant pour le retour des "Roms» en Roumanie ou en Bulgarie. Ces deux séquences politiques ont profondément ancré dans les représentations collectives l'association entre "Tsiganes de l'Est » et bidonville (Fassin et al., 2014; Olivera, 2011). Surtout, elles ont durci l'opinion de la société française à l'encontre de cette population. Ces changements se sont répercutés en profondeur dans leur rapport aux institutions et aux autres groupes sociaux.

Le lien à la société française des familles de "La place " dépend largement des ressources culturelles dont ils disposent et de la teneur des contacts qu'ils peuvent entretenir avec les autres habitants du quartier ou de l'agglomération (Lapeyronnie, 2008). Ainsi, l'apprentissage $d u$ français facilite l'interaction avec l'extérieur et l'acquisition des codes ou des normes, rendant également plus aisée la négociation du stigmate qu'ils endossent. C'est particulièrement le cas des plus jeunes qui parlent couramment le français et qui disposent du plus de liens à l'extérieur. Un certain nombre d'entre eux ont ainsi établi des relations consolidées avec plusieurs communautés du quartier en se rattachant partiellement à eux. C'est ce que vit par exemple Adi, père de famille dont nous avons déjà évoqué le parcours un peu plus haut. Lui et son frère se sont intégrés à un groupe de jeunes hommes de leur âge dont la majorité sont d'origine algérienne. Pour eux, d'une certaine façon, ils se sentent « plus Arabes que Roumains maintenant. À force de trainer avec eux, c'est normal ». Cette relation particulière avec les autres citadins ethnicisés est loin d'être généralisable aux autres membres du bidonville dont certains subissent encore une forte stigmatisation dans le quartier (insultes, menaces, etc.), choses auxquelles ils ne peuvent pas répondre dès lors qu'ils ne parlent pas le français ou ne maîtrisent pas les codes nécessaires à la négociation.

On retrouve ce type d'interactions ambivalentes lors des relations entre les habitants de «La place» et les «manouches » de l'aire d'accueil des gens du voyage située à quelques centaines de mètres du bidonville. Alors que, grâce à l'évangélisme ou au 
métier de ferrailleur, plusieurs pères de famille entretiennent des relations solides avec les membres de cette communauté, d'autres habitants, ceux parlant généralement le moins français, restent relativement en marge, suscitant d'ailleurs plus que les autres la méfiance des autres communautés tsiganes. C'est ce qu'illustre un conflit à deux doigts de s'envenimer entre gens de "La place » et ceux de l'aire d'accueil lorsque Camelia, doyenne du bidonville dont nous avons déjà évoqué les conditions de vie un peu plus haut, s'est vue accusée par une femme âgée de l'aire d'accueil de l'avoir volée lors du marché dominical. Finalement, l'intervention des évangélistes des deux groupes a permis de dissiper le malentendu. Après de longues discussions, ils réussissent finalement à montrer que les responsables étaient probablement un autre groupe Tsigane, censé être bien moins respectable que les fidèles croyants et pratiquants réunis sur le bidonville à ce moment-là.

Ce rapport disparate à la société française s'observe également à travers la relation aux institutions, aux bénévoles associatifs et aux travailleurs sociaux. Sur le bidonville de "La place », les familles sont amenées à en côtoyer près d'une dizaine. Là aussi, les affinités dépendent souvent des ressources dont disposent les habitants pour communiquer ainsi que trouver des points d'achoppement ouvrant la voie à des relations plus personnalisées. Si les acteurs de terrain se donnent généralement comme objectif de proposer leur aide aux familles de façon indifférenciée, le plus souvent des liens privilégiés se tissent avec certains habitants, facilitant l'acquisition par ces derniers des codes de l'aide sociale ou même l'accès à des dispositifs d'accompagnement. En outre, près de la moitié des pères et mères de famille ont des enfants scolarisés. Ils sont ainsi mis en contact régulièrement avec les représentants de l'Éducation nationale qui participent à les socialiser aux institutions de l'État, tout en les confrontant également à certaines formes de violence symbolique (Dubet et Lapeyronnie, 1999 ; Zanten, 2012) desquelles restent éloignés les autres habitants.

31 Au-delà des travailleurs sociaux, les contacts dont disposent les habitants de «La place» sont des ressources importantes pour faciliter leur intégration au sein du système de protection sociale. Ceci permet d'expliquer que certaines familles disposent d'appartements leur permettant d'obtenir des allocations, notamment grâce à plusieurs connaissances ayant trouvé le logement. Ces dernières se sont portées garantes et se sont occupées des démarches administratives auprès de la Caisse d'allocations familiales (CAF). Ainsi, au quotidien, la maîtrise différenciée des normes et des compétences spécifiques à la vie en France permet d'identifier plusieurs formes d'expériences de la vulnérabilité au sein du bidonville. Tandis qu'une partie des habitants sont fortement exposés au racisme et à la marginalisation, d'autres, au contraire, bénéficient de ressources leur donnant accès à une place au sein de la société moins marquée par l'exclusion.

\section{Conclusion}

Sur au moins quatre points, il est possible de montrer en quoi les expériences de la vulnérabilité dans le bidonville sont plus complexes qu'il n'y paraît, et qu'elles mènent à des situations souvent très contrastées. Certaines familles peuvent être confrontées à une importante fragilité économique, mais disposent au contraire de conditions d'habitat plus favorables. D'une autre manière, des habitants peuvent avoir développé des liens privilégiés au sein de l'agglomération mais s'inscrire dans des parcours de 
rupture avec leur pays d'origine, faisant du bidonville le seul lieu de vie auquel se raccrocher. Dès lors, il s'agit de bien différencier les situations individuelles pour mieux comprendre en quoi le bidonville constitue effectivement une épreuve de précarité que les individus amortissent de façon variable selon leur situation de fragilité. Si dans chaque cas les protections déployées par les habitants face à la vulnérabilité représentent peu de choses, ces réponses peuvent toutefois être profondément différentes les unes des autres.

Pour autant, bien que le bidonville mette en scène des situations individuelles, il ne s'agit pas d'oublier que les habitants bénéficient, au moins sous certains aspects, de la protection collective. En effet, les familles «mutualisent des ressources et des compétences précaires » (Bouillon, 2009) pour faire face ensemble à la fragilité de leur situation sociale. Or, si chacun érige des remparts pour se prémunir de la vulnérabilité, ces boucliers sont en partie partagés et lient les habitants les uns aux autres. Dès lors, il reste important de saisir les parcours sociaux à la lumière des expériences collectives et de ce qui lie entre eux les habitants.

\section{BIBLIOGRAPHIE}

Agier M. (2013), Campement urbain, Paris, Payot \& Rivages.

Bouillon F. (2009), Les mondes du squat, Paris, PUF.

Bourgois L., Le Cleve A., Masson-Diez É. et Peyroux O. (2015), « Du bidonville à la ville », rapport de recherche, Association TRAJECTOIRES.

Certeau M. de (1990), L'invention du quotidien. 1. Arts de faire, Paris, Gallimard.

Damon J. (2017), Un monde de bidonvilles, Paris, Seuil.

Délégation interministérielle à l'hébergement et à l'accès au logement (2017), « $10^{\mathrm{e}}$ état des lieux national des campements illicites et bidonvilles », rapport pour la Délégation interministérielle à l'hébergement et à l'accès au logement.

Dubet F. et Lapeyronnie D. (1999), Les quartiers d'exil, Paris, Seuil.

Fassin É., Fouteau C., Guichard S. et Windels A. (2014), Roms et riverains, Paris, La Fabrique.

Fondation Abbé Pierre (2018), « $23^{\mathrm{e}}$ rapport sur l'état du mal-logement en France », rapport pour la Fondation Abbé Pierre.

Gaulejac V. de, Blondel F. et Taboada-Leonetti M. I. (2014), La lutte des places, Paris, Desclée De Brouwer.

Girola C. M. (2011), Vivre sans abri, Paris, Éditions Rue d'Ulm.

Lapeyronnie D. (2008), Ghetto Urbain, Paris, Robert Laffont.

Lion G. (2015), Incertaines demeures, Montrouge, Bayard.

Lussault M. (2009), De la lutte des classes à la lutte des places, Paris, Grasset. 
Martuccelli D. (2006), Forgé par l'épreuve, Paris, Armand Colin.

Martuccelli D. (2010), La société singulariste, Paris, Armand Colin.

Olivera M. (2011), Rom en (bidon)villes, Paris, Éditions Rue d'Ulm.

Pichon P. (2010), Vivre dans la rue, Saint-Étienne, Presses universitaires de Saint-Étienne.

Potot S. (2007), Vivre à l'Est, travailler à l'Ouest, Paris, L'Harmattan.

Renahy N. (2010), « Classes populaires et capital d'autochtonie. Genèse et usage d'une notion. », Regards Sociologiques, $\mathrm{n}^{\circ} 40$.

Retière J.-N. (2003), « Autour de l'autochtonie. Réflexions sur la notion de capital social populaire ", Politix, nº 63, p. 121-143.

Sayad A. (2014), La double absence, Paris, Seuil.

Scott J. C. (2009), La Domination et les arts de la résistance, Paris, Éditions Amsterdam.

Weber F. (2009), Le travail à-côté, Paris, EHESS.

Zanten A. VAN (2012), L'école de la périphérie, Paris, PUF.

\section{RÉSUMÉS}

Au cours des dernières décennies, les vulnérabilités résidentielles ont connu un nouvel essor en France, au point de faire dire à la Fondation Abbé Pierre qu'il y aurait 15 millions de personnes confrontées à des difficultés liées au logement en France. Cette situation peut néanmoins être observée à partir de prismes multiples. Parmi ceux-ci, les bidonvilles incarnent probablement la forme la plus médiatique. Ce type de logement (habitat informel, léger et regroupant au minimum plusieurs dizaines d'habitants) est généralement considéré comme le résultat de contraintes structurelles plurielles pesant sur leurs occupants. Pour autant, les modalités sociales, architecturales et spatiales que donnent à voir les bidonvilles ne sont pas du simple ressort des déterminants extérieurs, mais découlent également des caractéristiques des parcours migratoires des habitants, de leurs activités économiques, de leurs ressources disponibles pour améliorer l'habitat ou encore de leurs relations avec les institutions et les personnes extérieures du quartier. Ainsi, cet article propose d'interroger les formes multiples de l'expérience vécue de la vulnérabilité en fonction des différentes dimensions qui distinguent les vies quotidiennes des habitants des bidonvilles.

In recent decades, residential vulnerabilities have experienced a new boom. This to the point of making the Abbé Pierre foundation say that they would be 15 million people to be confronted with difficulties related to housing. This situation can be observed from multiple prisms. Among them, slums are probably the most media-intensive form. This type of housing (informal, light housing with at least several dozen inhabitants) is generally considered as the result of multiple structural constraints weighing on their occupants. Nevertheless, the spatial, architectural and spatial modalities taken by slums are not simply the result of external determinants, but also of interstices and resources invested by individuals to mitigate the impact that vulnerabilities can have. Thus, this article proposes to question how slum dwellers shape their situation of residential fragility through their collective investment in a place to live. 
INDEX

Mots-clés : vulnérabilités résidentielles, bidonvilles, microrésistances, chez-soi, protection collective

Keywords : residential vulnerabilities, slums, micro-resistance, home, collective protection

\section{AUTEUR}

JEAN-BAPTISTE DAUBEUF

Université de Lorraine, 2L2S 